\title{
Fair Adaptive Power Allocation for Cell-Free Massive MIMO Systems
}

\author{
Juno V. Saraiva, Roberto P. Antonioli, Gábor Fodor, F. Rafael M. Lima, Walter C. Freitas Jr. and Yuri C. B. Silva
}

In this context, several significant optimization problems have been investigated with different purposes. For example, subject to per-user QoS constraints and assuming conjugate beamforming precoder design, different strategies to minimize power consumption were addressed in [2]-[4]. However, unlike [3] and [4], the authors in [2] also investigated other objective functions, such as maximizing the sum of the signal to interference-plus-noise ratio (SINR). Although minimizing the power consumption is important from the energy efficiency point of view, it does not guarantee a fair resource allocation.

Taking into account fairness issues, approaches such as the max-min power control problem that inherently leads to uniform service provision to all users were discussed in [5]-[7]. Specifically, the downlink performance of cellfree massive MIMO systems was studied in [5] in terms of maximizing the minimum data rate among all users. Within this framework, the authors assumed and compared the wellknown conjugate beamforming and zero-forcing precoders, and thereby efficient iterative solutions based on the bisection method were proposed. Analogously, the max-min power control problem was also considered with conjugate beamforming in [6], where the authors investigated both downlink and uplink cases. Iterative solutions via the bisection method were also proposed therein. The authors compared the performance of cell-free systems and architectures based on traditional cells, particularly small-cell networks and showed that cell-free massive MIMO systems can significantly outperform smallcell systems in terms of throughput. Furthermore, it was shown that cell-free systems are much more robust to shadow fading correlation than small-cell systems.

In [7], differently from [5] and [6], the max-min fairness problem was addressed also taking into account transceiver hardware impairments. Interestingly, therein the authors proved that cell-free massive MIMO can tolerate hardware impairments without performance degradation. Given the relevance of the topic of fairness-driven resource allocation, recent works focused on the max-min problem to investigate the performance of cell-free systems from different perspectives [8] [12]. In particular, the effects of the users in the system and antennas at APs on the spectral efficiency (SE) were analyzed through the use of max-min fairness power control in [8], while in [9] the objective of designing the optimum beamforming for maximizing the minimum instantaneous SINR of all users was considered. Meanwhile, [10] proposed two fully distributed precoding schemes and quantitatively compared them through the max-min fairness power control. Precoding schemes were also studied in [11], but, in that case, variants of conjugate beamforming design were proposed, analyzed and compared in the context of max-min fairness control. In [12], 
the authors addressed the max-min SE and sum-SE power allocation policies and two new algorithms were proposed for sum-SE power optimization inspired by weighted minimum mean square error (WMMSE) minimization and fractional programming. Moreover, for max-min fairness power allocation, as an alternative to the bisection method, a new iterative solution based on fractional programming was also proposed.

Cell-free massive MIMO systems have been extensively discussed in [1] taking into account several aspects. Therein, important problems such as the SE maximization and minimum SINR maximization problems were widely investigated. In particular, in addition to the bisection method, for the problem of maximizing the minimum SINR, another efficient iterative approach was proposed. Similar to the bisection method, that strategy has low computational cost and quickly converges, but, interestingly, it does not depend on successive solutions of parametric problems.

Inspired by the above discussion, this paper considers the downlink of a cell-free massive MIMO system where the APs employ conjugate beamforming. We focus on max-min fairness power control algorithms that maximize the minimum SINR among all users. Unlike common solutions found in the literature, we propose a non-iterative power allocation policy. Interestingly, by adjusting a scalar parameter, the proposed solution can provide different trade-offs between fairness and total SE in the system. Computational simulations corroborate that the trade-off between fairness and total SE can be effectively controlled by using a scalar parameter and that the proposed solution has a near-optimal performance in terms of maximizing the minimum SINR.

\section{System Model}

We consider the downlink of cell-free massive MIMO systems, where $N$ APs arbitrarily distributed over the coverage area coherently serve $K$ users and $N \gg K$, as illustrated in Figure 1. All APs and users are equipped with a single antenna and grouped in sets $\mathcal{N}$ and $\mathcal{K}$, respectively. We adopt conjugate beamforming, which has a low complexity cost since it is done locally and no channel state information (CSI) exchange among the APs is required. Furthermore, we assume that the APs are connected via backhaul links to edgecloud processors, called CPUs that can perform large-stage processing tasks to detect or decode the signals of the users. Although backhaul links may be subject to significant practical constraints, such analysis is outside of the scope of this paper and, thus, we consider error-free backhaul links.

As it is typically done in massive MIMO, we consider the time division duplexing (TDD) protocol, in which a first phase is dedicated for channel estimation (via uplink training) and the second phase is used for downlink data transmission [6]. Therefore, after channel estimation, the APs can use the obtained CSI for data transmission relying on channel reciprocity. Each coherence block is divided into $\tau_{\mathrm{p}}$ channel uses for uplink training followed by $\tau_{\mathrm{d}}$ channel uses for downlink transmission such that $\tau_{\mathrm{c}}=\tau_{\mathrm{p}}+\tau_{\mathrm{d}}$, where $\tau_{\mathrm{c}}$ is the coherence block length in channel uses. We assume, without loss of generality, that the number of users is $K \leq \tau_{\mathrm{p}}$, i.e., the pilot sequences are

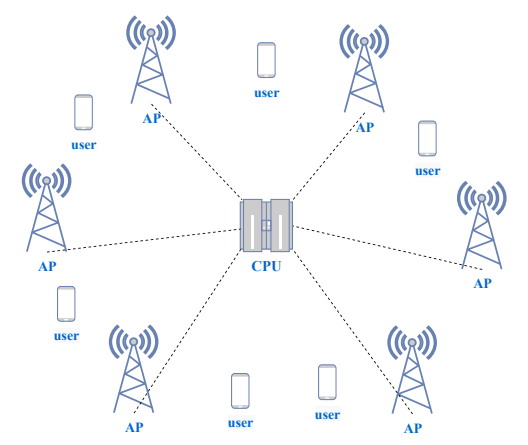

Fig. 1: Illustration of a cell-free massive MIMO system assuming $N \gg K$.

created such that they are orthogonal until the number of users in the system is maximum $\tau_{\mathrm{p}}$. Thereby, we admit an orthogonal assignment model, where each user can get assigned a pilot sequence mutually orthogonal to the others.

\section{A. Channel Model}

The channel gain between APs and the users are represented by a complex matrix $\mathbf{G}=\left[\mathbf{g}_{1}, \mathbf{g}_{2}, \ldots, \mathbf{g}_{K}\right]$, where $\mathbf{g}_{k}=$ $\left[g_{1, k}, \ldots, g_{N, k}\right]^{\mathrm{T}} \in \mathbb{C}^{N \times 1}$ is the collective channel vector from all APs to user $k$. The channel coefficient between AP $n$ and user $k$ is modelled as follows [4], [6]:

$$
g_{n, k}=\beta_{n, k}^{1 / 2} h_{n, k}, \quad \forall n \in \mathcal{N} \text { and } \forall k \in \mathcal{K},
$$

where $h_{n, k}$ corresponds to small-scale fading and $\beta_{n, k}$ represents the large-scale propagation losses, including path loss and shadowing. We assume that $\left\{h_{n, k}\right\} \forall n, k$ are independent and identically distributed $\mathcal{C N}(0,1)$.

\section{B. Uplink Pilot-Based Channel Estimation}

As briefly discussed earlier, before transmitting data, i.e., during the training phase and in a distributed way, each AP acquires CSI knowledge via uplink pilot-assisted channel estimation. Typically, the communication in TDD-based cellfree massive MIMO networks starts with the users simultaneously sending known orthogonal pilot sequences to allow the channels to be estimated at each AP. Assuming synchronization across the network, the received uplink signal at AP $n$, corresponding to the pilot transmissions is given by [6]

$$
\mathbf{y}_{n}^{\text {pilot }}=\sqrt{\tau_{\mathrm{p}} \rho_{\mathrm{p}}} \sum_{k \in \mathcal{K}} g_{n, k} \boldsymbol{\varphi}_{k}+\mathbf{n}, \quad \forall n \in \mathcal{N},
$$

where $\varphi_{\boldsymbol{k}}$, with $\left\|\boldsymbol{\varphi}_{\boldsymbol{k}}\right\|^{2}=1$, denotes the $\tau_{\mathrm{p}} \times 1$ orthogonal pilot sequence assigned to user $k, \rho_{\mathrm{p}}$ is the pilot's transmit power and $\mathbf{n} \in \mathbb{C}^{\tau_{\mathrm{p}} \times 1}$ is the Gaussian noise vector whose elements follow $\mathcal{C N}\left(0, \sigma^{2}\right)$, in which $\sigma^{2}$ is the noise power.

In the context of cell-free systems, different approaches can be employed at the APs for channel estimation. When channel estimation is based on minimizing the mean squared error (MSE) among all possible estimations, the minimum mean squared error (MMSE) estimate can be obtained as presented in the following result [6]: 
Corollary 1: The MMSE estimate of $g_{n, k}$, i.e., $\left\{\hat{g}_{n, k}\right\} \forall n, k$ based on the received pilot signal at AP $n$ is:

$$
\hat{g}_{n, k}\left(\mathbf{y}_{n}^{\text {pilot }}\right)=\frac{\sqrt{\tau_{\mathrm{p}} \rho_{\mathrm{p}}} \beta_{n, k}}{\tau_{\mathrm{p}} \rho_{\mathrm{p}} \sum_{i \in \mathcal{K}} \beta_{n, i}\left|\boldsymbol{\varphi}_{k}^{\mathrm{H}} \boldsymbol{\varphi}_{\boldsymbol{i}}\right|^{2}+\sigma^{2}} \boldsymbol{\varphi}_{k}^{\mathrm{H}} \mathbf{y}_{n}^{\text {pilot }} .
$$

\section{SINR and Achievable SE}

Assuming that conjugate beamforming is employed in the downlink, the received SINR at user $k$ is expressed as [4]:

$$
\operatorname{SINR}_{k}(\boldsymbol{\rho})=\frac{\eta^{2} \rho_{k}\left|\hat{\mathbf{g}}_{k}^{\mathrm{H}} \mathbf{g}_{k}\right|^{2}}{\sum_{i \in \mathcal{K} \backslash\{k\}} \eta^{2} \rho_{i}\left|\hat{\mathbf{g}}_{k}^{\mathrm{H}} \mathbf{g}_{i}\right|^{2}+\sigma^{2}}, \quad \forall k \in \mathcal{K},
$$

where $\eta=\left\|\hat{\mathbf{G}}^{\mathrm{H}}\right\|_{\mathrm{F}}^{-1}$ is the normalization factor and $\boldsymbol{\rho}=$ $\left[\rho_{1}, \ldots, \rho_{K}\right]^{\mathrm{T}}$ is the vector of transmit power coefficients for all the $K$ users. The transmit powers are non-negative, i.e., $\rho_{k} \geq 0, \forall k \in \mathcal{K}$, represented by $\boldsymbol{\rho} \succeq \mathbf{0}_{K}$. Based on the definition of SINR in (4), we can also define the SE expression for a given user $k$ according to the following result [1]:

Theorem 1: The achievable SE of user $k$ in the downlink is given by

$$
\operatorname{SE}_{k}(\boldsymbol{\rho})=\frac{\tau_{\mathrm{d}}}{\tau_{\mathrm{c}}} \log _{2}\left(1+\operatorname{SINR}_{k}(\boldsymbol{\rho})\right), \quad[\mathrm{bit} / \mathrm{s} / \mathrm{Hz}] .
$$

\section{Problem Formulation}

Aiming at obtaining uniform and good service to all users based on the achieved SINR, we formulate the max-min power control problem under per-AP power constraints as:

$$
\begin{aligned}
& \underset{\boldsymbol{\rho} \succeq \mathbf{0}_{K}}{\operatorname{maximize}} \min _{k \in \mathcal{K}} \operatorname{SINR}_{k}(\boldsymbol{\rho}) \\
& \text { subject to } \mathbf{a}_{n}^{\mathrm{T}} \boldsymbol{\rho} \leq \rho_{\max }, \quad \forall n \in \mathcal{N},
\end{aligned}
$$

where the fixed vector $\mathbf{a}_{n} \in \mathbb{R}_{>0}^{K}, \forall n \in \mathcal{N}$ specifies the weighting coefficient for each user's power coefficient and $\rho_{\max }$ is the power budget of each AP. In summary, the aim in (6) is to find the optimal power allocation vector, $\boldsymbol{\rho}^{\star}$, which maximizes the lowest SINR among all users in the network. At this optimum point, all users achieve the same SINR value.

\section{Proposed Solutions}

\section{A. Optimal Solutions Based on Iterative Optimization}

Based on the SINR structure, a fixed-point algorithm can be implemented to obtain the optimal solution of problem (6) as proposed in [1]. In this case, the SINR expression should satisfy certain additional conditions as stated in Lemma 1 [1].

Lemma 1: Suppose that $\left\{\operatorname{SINR}_{k}(\boldsymbol{\rho})\right\}_{\forall k}$ satisfy the following conditions:

1) $\operatorname{SINR}_{k}(\boldsymbol{\rho})>0$ if $\boldsymbol{\rho} \geq \mathbf{0}_{K}$ and $\operatorname{SINR}_{k}(\boldsymbol{\rho})=0$ if and only if $\rho_{k}=0, \forall k \in \mathcal{K}$;

2) $\operatorname{SINR}_{k}(\boldsymbol{\rho})$ is strictly increasing with respect to $\rho_{k}$ and is strictly decreasing with respect to $\rho_{i}$, for $i \neq k$, when $\rho_{k}>0, \forall k \in \mathcal{K}$

3) For $\lambda>1, \rho_{k}>0, \operatorname{SINR}_{k}(\lambda \boldsymbol{\rho})>\operatorname{SINR}_{k}(\boldsymbol{\rho}), \forall k \in \mathcal{K}$;

Clearly, the SINR expression in (4) complies with Lemma 1 and, thereby, Algorithm 1 can be used to optimally solve

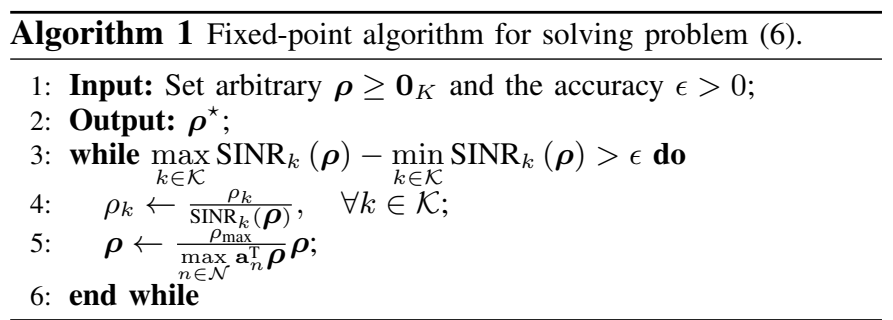

problem (6) [1]. Particularly, Algorithm 1 is interesting because it does not depend on successive solutions of other problems to obtain an optimal solution to problem (6). Besides, it quickly converges and has low computational cost since it only involves iterative closed-form updates of the variables.

Indeed, it is possible to explore other strategies to achieve the optimal solution of problem (6). However, although these other strategies are based on more generic approaches, in general, they depend on solutions of parametric problems. In this context, first note that problem (6) can be expressed in the epigraph form as

$$
\begin{aligned}
& \underset{\boldsymbol{\rho} \in \boldsymbol{\Omega}, \varepsilon \in \mathbb{R}_{+}^{*}}{\operatorname{maximize}} \varepsilon \\
& \text { subject to } \frac{f_{k}(\boldsymbol{\rho})}{g_{k}(\boldsymbol{\rho})} \geq \varepsilon, \quad \forall k \in \mathcal{K},
\end{aligned}
$$

where set $\Omega$ is the optimization domain or the solution space of problem (6), and $f_{k}$ and $g_{k}$ are continuous, affine, differentiable and real-valued functions of $\rho \in \Omega$ and consist in the numerator and denominator of the SINR expression in (4), respectively. Due to the considered constraints, $\Omega$ is a compact and nonempty set.

However, the formulation in (7) is not jointly convex in $\rho$ and $\varepsilon$. Nevertheless, for a fixed value of $\varepsilon$, we obtain a linear programming (LP) problem and, furthermore, we arrive at a classical feasibility problem in $\rho$ that can be optimally solved by the classical bisection method. As an alternative, we can rearrange constraint ( $7 \mathrm{~b})$ and obtain:

$$
f_{k}(\boldsymbol{\rho})-\varepsilon g_{k}(\boldsymbol{\rho}) \geq 0, \quad \forall k \in \mathcal{K} .
$$

Therefore, note that problem (7) is feasible if

$$
F(\varepsilon)=\max _{\boldsymbol{\rho} \in \boldsymbol{\Omega}} \min _{k \in \mathcal{K}}\left\{f_{k}(\boldsymbol{\rho})-\varepsilon g_{k}(\boldsymbol{\rho})\right\} \geq 0 .
$$

From the generalized fractional programming theory [14], there is a powerful relationship between the optimal value of parameter $\varepsilon$, i.e., $\varepsilon^{\star}$, and $F(\varepsilon)$ as stated in Theorem 2 [14]:

Theorem 2: $\varepsilon^{\star}=\min _{k \in \mathcal{K}} \frac{f_{k}\left(\boldsymbol{\rho}^{\star}\right)}{g_{k}\left(\boldsymbol{\rho}^{\star}\right)}=\max _{\boldsymbol{\rho} \in \boldsymbol{\Omega}}\left\{\min _{k \in \mathcal{K}} \frac{f_{k}(\boldsymbol{\rho})}{g_{k}(\boldsymbol{\rho})}\right\}$, where $\boldsymbol{\rho}^{\star} \in \boldsymbol{\Omega}$ solves problem (7) if and only if

$$
F\left(\varepsilon^{\star}\right)=\max _{\boldsymbol{\rho} \in \boldsymbol{\Omega}} \min _{k \in \mathcal{K}}\left\{f_{k}(\boldsymbol{\rho})-\varepsilon^{\star} g_{k}(\boldsymbol{\rho})\right\}=0 .
$$

Briefly, Theorem 2 states that solving the original problem, i.e., problem (6), is equivalent to finding the root of function $F(\varepsilon)$ defined in (9). Many iterative algorithms can be employed for finding the root of $F(\varepsilon)$. Among these algorithms, we highlight the Dinkelbach's approach, which is based on the application of Newton's method. In general, the Dinkelbach's approach is quite efficient because the sequence converges to the optimal point with a superlinear convergence rate [14]. 


\section{B. Alternative Solutions Based on Linear Optimization}

Fundamentally, resource allocation solutions to provide fairness among users should inherently direct efforts towards the users with low SINR values. Certainly, the use of this strategy must also be taken into account in any effective sub-optimal solution. In this sense, to develop sub-optimal solutions for problem (6), we first classify all users of the system according to the following criterion:

$$
\varrho_{k}=\frac{\eta^{2}\left|\hat{\mathbf{g}}_{k}^{\mathrm{H}} \mathbf{g}_{k}\right|^{2}}{\sum_{i \in \mathcal{K} \backslash\{k\}} \eta^{2}\left|\hat{\mathbf{g}}_{k}^{\mathrm{H}} \mathbf{g}_{i}\right|^{2}+\sigma^{2}}, \quad \forall k \in \mathcal{K} .
$$

By exploiting the definition of the SINR in (4), the values $\left\{\varrho_{k}\right\}_{\forall k}$ defined in (11) act as an important metric to classify the users regarding their difficulties in obtaining SINR gains in power-constrained systems. More specifically, the lower the value of $\varrho_{k}$ for a given user $k$, the more costly it is for the system, in terms of used resources, to increase its SINR. Therefore, from the point of view of the achieved SINR, the user with the lowest $\varrho_{k}$ value is the worst user of the system.

Through the definition in (11), we can develop a resource allocation policy to direct power to the users with the greatest difficulties in obtaining SINR gains. This strategy depends on the following optimization problem:

$$
\begin{aligned}
& \underset{\boldsymbol{\rho} \geq \mathbf{0}_{K}}{\operatorname{maximize}} \min _{k \in \mathcal{K}} \rho_{k}\left(\varrho_{k}\right)^{\theta} \\
& \text { subject to } \mathbf{a}_{n}^{\mathrm{T}} \boldsymbol{\rho} \leq \rho_{\max }, \quad \forall n \in \mathcal{N},
\end{aligned}
$$

where $\theta \in \mathbb{R}$ is a fixed parameter, which can change the user priority on the resource allocation as described next.

Specifically, in problem (12) a higher value of $\theta$ tends to allocate more power to users with a lower value of $\varrho_{k}$. Consequently, $\theta$ is an important parameter for the system as it may lead to different levels of fairness in terms of SINR floor. This is interesting because the choice of $\theta$ can be exploited in order to achieve other important goals in the context of mobile networks. Particularly, by adjusting $\theta$, it is possible to allocate resources to improve SE at the cost of higher unfairness in the system. Unlike previous solutions, for a given value of $\theta$, our sub-optimal solution to problem (6) is obtained in a single instance, i.e., it is not iterative. For this, note that problem (12) can be written in epigraph form as an LP problem as follows:

$$
\begin{aligned}
& \underset{\boldsymbol{\rho} \in \boldsymbol{\Omega}, \xi \in \mathbb{R}_{+}^{*}}{\operatorname{maximize}} \xi \\
& \text { subject to } \rho_{k}\left(\varrho_{k}\right)^{\theta} \geq \xi, \quad \forall k \in \mathcal{K} .
\end{aligned}
$$

Therefore, given a fixed value $\theta$, a sub-optimal solution of problem (6) can be obtained in a single instance through the solution of problem (13).

However, instead of setting a specific initial value of $\theta$, we can also exploit an iterative procedure to provide a better solution for problem (6) through successive solutions of problem (13) as shown in Algorithm 2. Algorithm 2 should be initialized with the set $\Theta$ containing all values of $\theta$ that must be employed to solve problem (13). By varying $\theta$, Algorithm 2 is able to show the impact of this parameter on

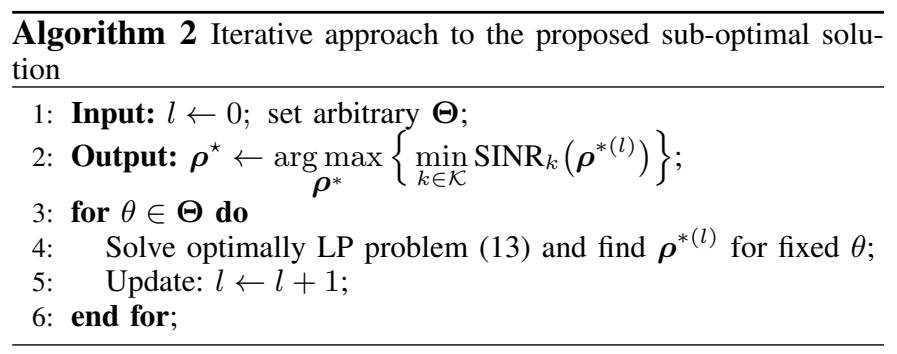

the performance of the solutions and thereby it addresses the important trade-offs that may be obtained in the system.

\section{Performance Evaluation}

In this section, we demonstrate via numerical results the effectiveness of the proposed sub-optimal power control design.

\section{A. Simulation Assumptions}

The setup assumed herein is based on the one introduced in [10]. In particular, the APs are located on a uniform grid in the area of $D \times D$, where $D=1 \mathrm{~km}$ and the height of each AP is of 10 meters. Meanwhile, the users are randomly distributed in this area. In order to avoid boundary effects, this square area is wrapped-around to imitate an infinite network environment. Moreover, we consider $N=100, K=10, \tau_{\mathrm{c}}=200, \tau_{\mathrm{p}}=10$, $\rho_{\mathrm{p}}=200 \mathrm{~mW}, \rho_{\max }=1 \mathrm{~W}$, and $20 \mathrm{MHz}$ bandwidth.

\section{B. Results and Discussions}

In Figure 2, we use Algorithm 2 to show the impact of the parameter $\theta$ on the performance of the obtained solutions in terms of the minimum SINR. To this end, we plot performance relative to the solution of two different random channel instances and vary the value of $\theta$ between 0 and 1 . It should be noted in this first figure that $\theta$ has an expressive influence on the solution of problem (13). Note that up to a certain value, increasing $\theta$ provides more power resources to the worst users and, consequently, minimum SINR is significantly enhanced. Indeed, this shows that the strategy adopted in Algorithm 2 is efficient in exploiting the parameter $\theta$ to provide fairness in the system in terms of SINR floor.

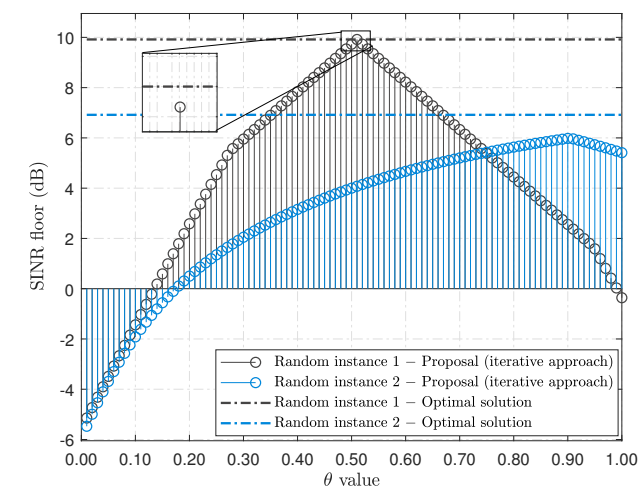

Fig. 2: SINR floor in dB versus $\theta$ value for the optimal and sub-optimal (by Algorithm 2) solutions considering two different random instances. 


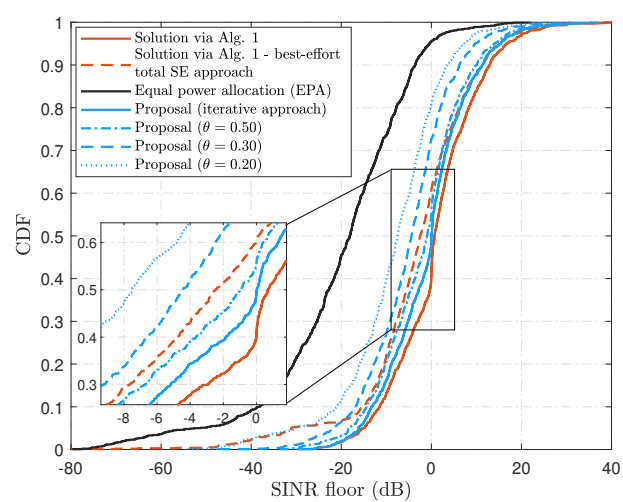

Fig. 3: CDFs of minimum SINR for the solutions of Algorithm 1, Proposal (considering Algorithm 2 and setting specific $\theta$ values) and EPA.

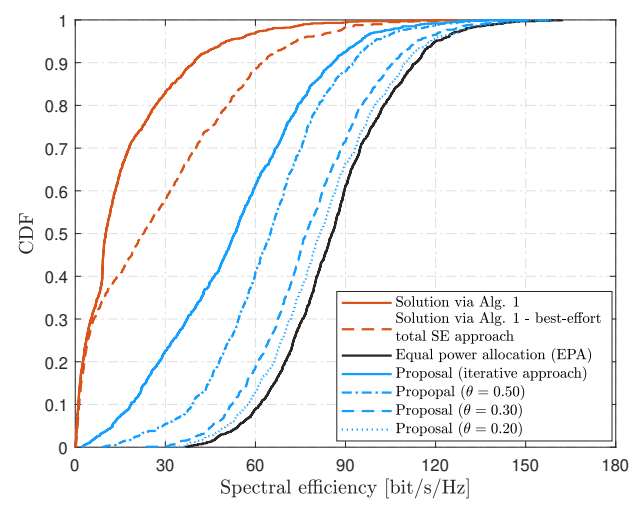

Fig. 4: CDFs of total SE for the solutions of Algorithm 1, Proposal (considering Algorithm 2 and setting specific $\theta$ values) and EPA.

Figures 3 and 4 show the CDFs of the SINR floor and total SE of the system, respectively. In these figures, we plot the solutions provided by Algorithm 1, our sub-optimal scheme proposed in Section IV-B and the equal power allocation (EPA) strategy. Specifically, regarding our sub-optimal solution, we consider two different approaches: one iterative approach employing Algorithm 2 and a non-iterative one considering specific $\theta$ values. Therefore, in Figures 3 and 4, there are four blue curves for our sub-optimal solution, i.e., three curves assuming three different specific values for $\theta$ $(0.20,0.30,0.50)$ and a last one assuming the solution found by Algorithm 2 with $\theta \in[0,1]$. For Algorithm 1 , beyond the solution to problem (6), we also consider the best that Algorithm 1 can provide in terms of total SE (dashed-red line).

Figure 3 shows that, as expected, the proposed sub-optimal solution is more efficient when used in its iterative form. However, when we take a mean value in the considered range, i.e., $\theta=0.50$, we have a small performance loss compared to the iterative version. Indeed, this is advantageous because for a specific $\theta$, the solution is obtained in a single instance using (13) and, thus, it has a lower computational cost. When $\theta<0.50$, from the SINR floor point of view, the solutions tend to deliver more unfair situations but with a higher total $\mathrm{SE}$, as shown in Figure 4. In this last figure, although we do not show the upper bound of the total SE, note that as we decrease $\theta$, our sub-optimal solution tends to approximate significantly to classical EPA solution, which is known to be a good benchmarking for maximizing the total SE. Lastly, mathematically note that case $\theta=0$, our sub-optimal solution converges exactly to the EPA solution. In general, these results show that, by adjusting $\theta$, our sub-optimal solution can provide different trade-offs of fairness and total SE.

\section{CONCLUSiOnS}

We analyzed the performance of cell-free massive MIMO systems taking into account the effects of channel estimation and power control design. In particular, we investigated power allocation policies to the classical max-min fairness problem. In this context, we initially presented different optimal strategies proposed in the literature to tackle this problem based on iterative optimization. Then, we proposed a novel sub-optimal approach that - unlike previous solutions - is not iterative. The proposed solution also allows for an iterative procedure, which can improve its performance. Through extensive computational simulations, we demonstrated that our power allocation policy is capable of providing different trade-offs between fairness among users and the total SE. Investigating scenarios with pilot contamination effect and proposing scalable power control strategies are interesting topics for future studies.

\section{REFERENCES}

[1] Özlem Tugfe Demir, E. Björnson, and L. Sanguinetti, "Foundations of user-centric cell-free massive MIMO," Foundations and Trends ${ }^{\circledR}$ in Signal Processing, vol. 14, no. 3-4, pp. 162-472, 2021.

[2] Y. Zhang, H. Cao, Y. Guo, and L. Yang, "SCA power optimization in cell-free massive MIMO with short-term power constraints," in 2018 10th International Conference on Wireless Communications and Signal Processing (WCSP), 2018, pp. 1-6.

[3] Y. Zhang, H. Cao, M. Zhou, Y. Li, and L. Yang, "Power minimization for cell-free massive MIMO," in 2019 IEEE International Conference on Consumer Electronics - Taiwan (ICCE-TW), 2019, pp. 1-2.

[4] R. Hamdi and M. Qaraqe, "Power allocation and cooperation in cellfree massive MIMO systems with energy exchange capabilities," in IEEE Vehicular Technology Conference (VTC) - Spring, May 2020, pp. 1-5.

[5] E. Nayebi, A. Ashikhmin, T. L. Marzetta, H. Yang, and B. D. Rao, "Precoding and power optimization in cell-free massive MIMO systems," IEEE Trans. Wireless Commun., vol. 16, no. 7, pp. 4445-4459, 2017.

[6] H. Q. Ngo, A. Ashikhmin, H. Yang, E. G. Larsson, and T. L. Marzetta, "Cell-free massive MIMO versus small cells," IEEE Trans. Wireless Commun., vol. 16, no. 3, pp. 1834-1850, March 2017.

[7] J. Zhang, Y. Wei, E. Björnson, Y. Han, and S. Jin, "Performance analysis and power control of cell-free massive MIMO systems with hardware impairments," IEEE Access, vol. 6, pp. 55 302-55314, 2018.

[8] T. C. Mai, H. Q. Ngo, and T. Q. Duong, "Downlink spectral efficiency of cell-free massive MIMO systems with multi-antenna users," IEEE Trans. Commun., vol. 68, no. 8, pp. 4803-4815, 2020.

[9] A. Zhou, J. Wu, E. G. Larsson, and P. Fan, "Max-min optimal beamforming for cell-free massive MIMO," IEEE Commun. Lett., vol. 24, no. 10 , pp. 2344-2348, 2020.

[10] G. Interdonato, M. Karlsson, E. Björnson, and E. G. Larsson, "Local partial zero-forcing precoding for cell-free massive MIMO," IEEE Trans. Wireless Commun., vol. 19, no. 7, pp. 4758-4774, 2020.

[11] G. Interdonato, H. Q. Ngo, and E. G. Larsson, "Enhanced normalized conjugate beamforming for cell-free massive MIMO," IEEE Trans. Commun., pp. 1-1, 2021.

[12] S. Chakraborty, O. T. Demir, E. Björnson, and P. Giselsson, "Efficient downlink power allocation algorithms for cell-free massive MIMO systems," IEEE Open Journal of the Communications Society, vol. 2, pp. 168-186, 2021.

[13] O. T. Demir and E. Björnson, "Joint power control and LSFD for wireless-powered cell-free massive MIMO," IEEE Trans. Wireless Commun., vol. 20, no. 3, pp. 1756-1769, 2021.

[14] J.-P. Crouzeix and J. A. Ferland, "Algorithms for generalized fractional programming," Mathematical Programming, vol. 52, no. 1-3, pp. 191207, 1991. 